\title{
Inter- and intra-observer agreement on Miller's classification of gingival tissue recessions
}

\author{
Kristina Bertl1,2, Dorothea Ruckenbauer ${ }^{1}$, Michael Müller-Kern ${ }^{3}$, Gerlinde Durstberger ${ }^{4}$, Stefan Lettner 5,6, Corinna Bruckmann4, Christian Ulm \\ 1 Division of Oral Surgery, Bernhard Gottlieb School of Dentistry, Medical University of Vienna, Austria \\ 2 Department of Periodontology, Faculty of Odontology, University of Malmö, Sweden \\ Academy of Oral Implantology, Vienna, Austria \\ ${ }^{4}$ Division of Conservative Dentistry and Periodontology, Bernhard Gottlieb School of Dentistry, Medical University of Vienna, Austria \\ ${ }^{5}$ Karl Donath Laboratory for Hard Tissue and Biomaterial Research, Division of Oral Surgery, Medical University of Vienna, Austria
}

${ }^{6}$ Austrian Cluster for Tissue Regeneration, Vienna, Austria

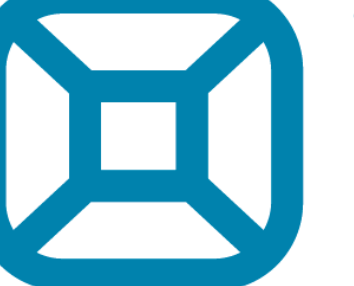

BERNHARD GOTTLIEB

UNIVERSITY CLINIC

OF DENTISTRY

\section{Purpose}

Miller's is the most commonly used classification of gingival tissue recessions (Miller 1985). However, data on the reliability of this classification are missing so far, although reliability, which reflects the consistency of repeated measurements, is regarded as a prerequisite for judging the utility of a classification (Karras 1997). The aim of the present study was to determine inter- and intra-observer agreement on Miller's classification and on 3 additional parameters associated with gingival tissue recessions.

\section{Methods}

Two hundred photographs (50 of each region: maxillary/mandibular anterior/posterior teeth) of gingival tissue recessions were evaluated twice (interval: one month) by 4 observers in Miller's classification (classes I to IV; Miller 1985), gingival phenotype (thin\&high or thick\&low scalloping; Seibert 1989), tooth shape (long-narrow or short-wide; Olsson 1991\&1993), and identifiability of the cemento-enamel junction (CEJ). The level of agreement was assessed according to a 6-level nomenclature (Landis 1977): poor $<0.0$, slight $0.0-0.2$, fair 0.21-0.4, moderate 0.41-0.6, substantial 0.61-0.8, and almost perfect 0.81-1.0.

\section{Results}

The inter- and intra-observer agreements on the assessed parameters are summarised in Table 1. The inter-observer agreement on Miller's classification was substantial, with the highest values for the anterior teeth. The intra-observer agreement was substantial to almost perfect, with the highest values for maxillary anterior teeth. The difference between the first and second ratings as well as among the different observers were mainly among Miller's classes I, II, and III, but never between classes I and IV (Figure 1 and 2).

The inter-observer agreement on the gingival phenotype was slight to moderate, with higher values for anterior mandibular teeth. Similar results were seen for intra-observer agreements. In general, the intra-observer agreements for all regions were moderate for each observer.

The inter-observer agreement on tooth shape was fair to moderate, with higher values for the anterior mandibular teeth. Similar results are presented for intra-observer agreement. In general, intra-observer agreement for all regions was moderate for each observer.

Inter-observer agreement on the identifiability of the CEJ was slight to fair, with values just slightly higher for anterior teeth. Intra-observer agreement was poor to almost perfect. The anterior mandibular teeth presented slightly higher values.
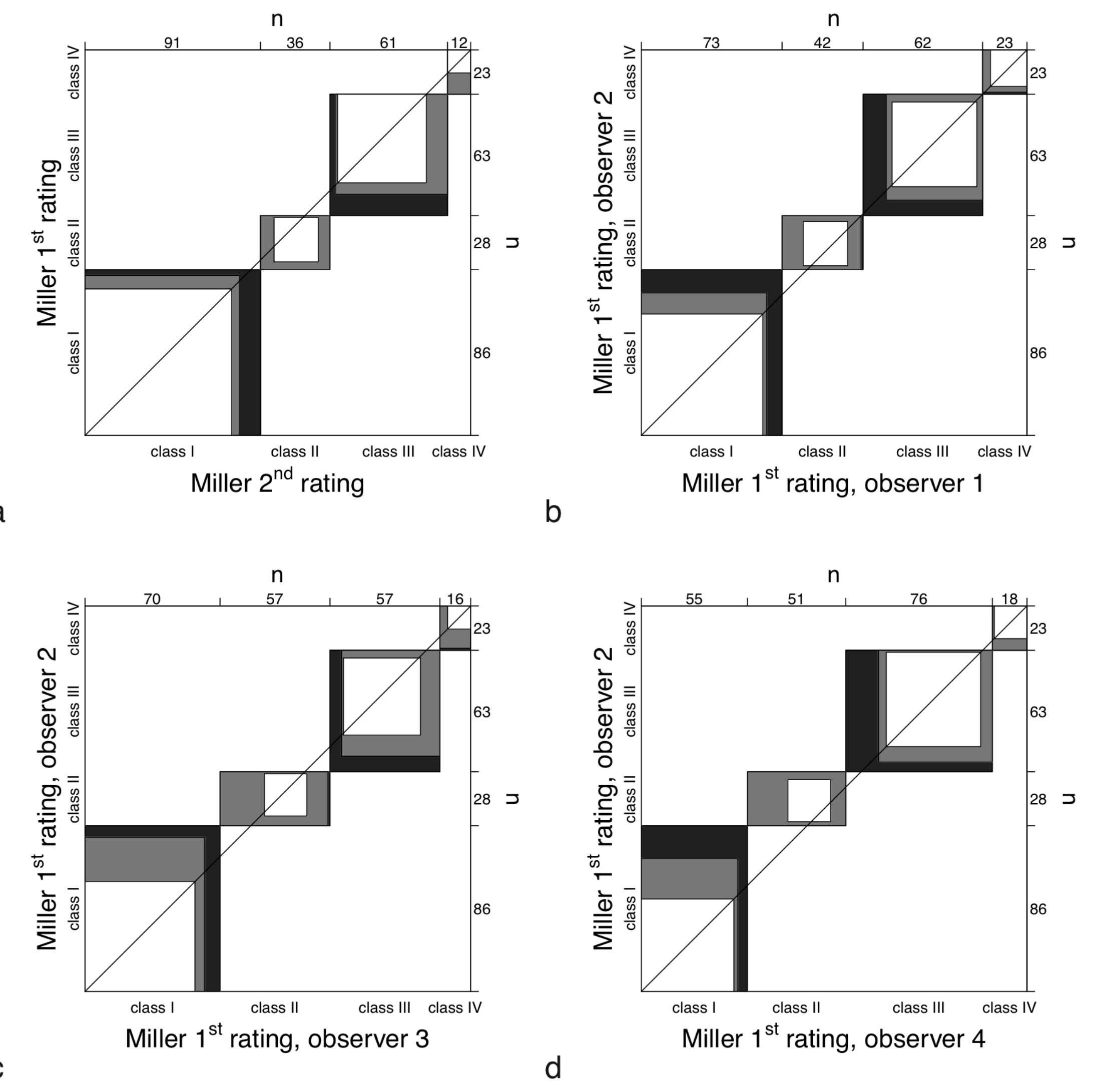

Figure 1. (a) Intra-observer agreement of Miller's classification for observer 2 and inter-observer agreement of Miller's classification for (b) observer 2 to 1 , (c) observer 2 to 3 , and (d) observer 2 to 4 . The white square represents a perfect match, the grey frame mismatch for one class, and the black frame mismatch for two classes. There was no mismatch for three classes (Miller's classes I vs. IV).
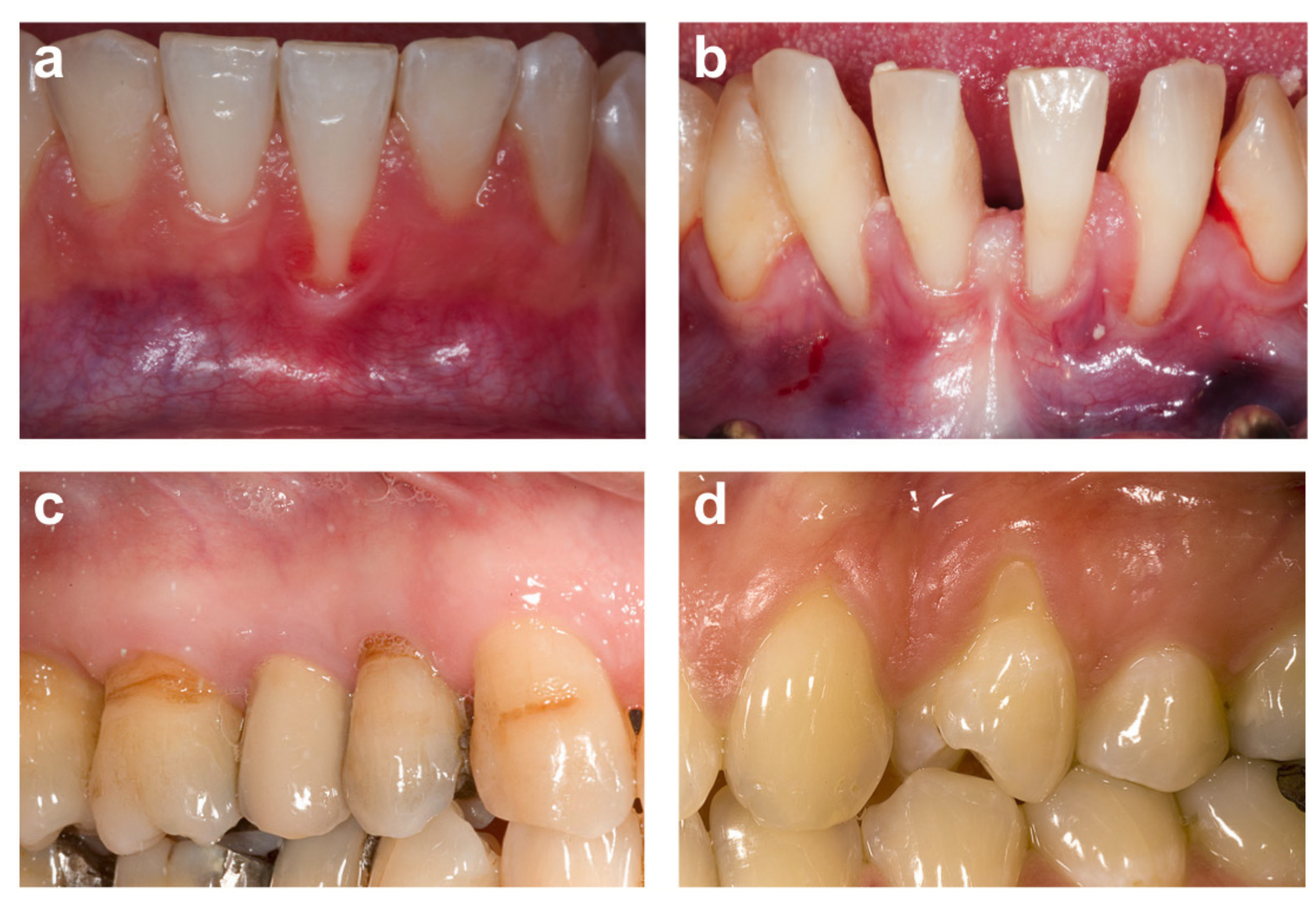

Figure 2. (a, b) Represent 2 teeth where all four observers agreed: (a) mandibular left central incisor, Miller class II; and (b) mandibular left central incisor, Miller class IV. (c, d) Represent 2 teeth where the four observers disagreed: (c) maxillary right first premolar, two said Miller class I and two said Miller class III; and (d) maxillary left first premolar, one said Miller class I, one Miller class II, and two Miller class III.

Table 1. Inter- and intra-observer agreements of the assessed parameters (Miller's classification, gingival phenotype, tooth shape, and identifiability of the CEJ).

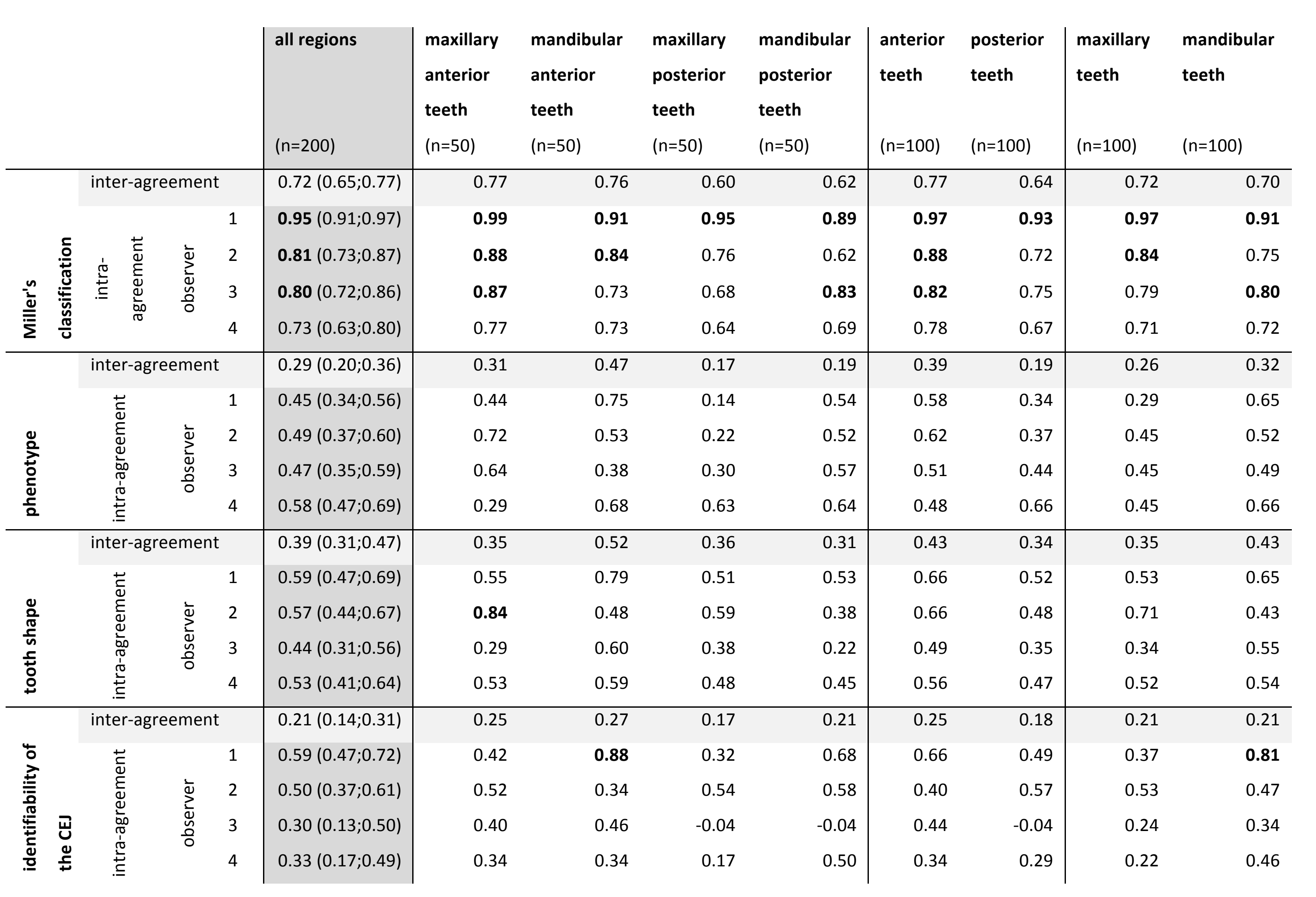

The inter-and intra-observer agreements of the Miller's classification were assessed by ICC and the other parameters by Kappas. In parentheses, the $95 \%$ Confidence Interval is presented for all regions. Parameters with an almost perfect reliability $(>0.80)$ are presented in bold. CEJ...cemento-enamel junction.

Gingival phenotype (thin\&high scalloping) significantly correlated with tooth shape (long-narrow) (rho=0.662, $p<0.001$; Table 2). Anterior teeth presented a higher correlation, with the mandibular anterior teeth presenting an almost perfect correlation (rho=0.954).

Table 2. Correlation between gingival phenotype and tooth shape (results from observer 2 , Spearman correlation coefficient) indicating an association between a thin and highscalloping gingival phenotype and long, narrow teeth.

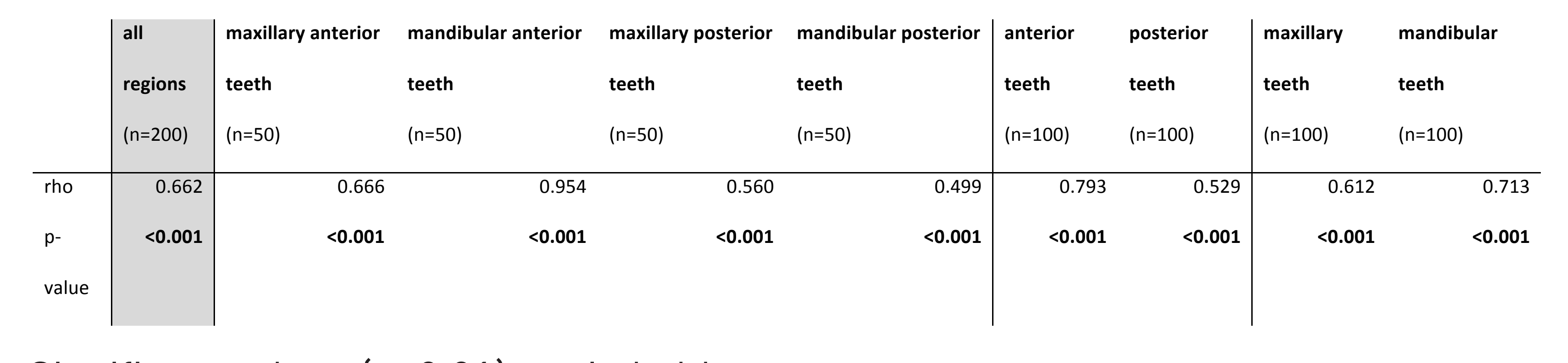

Significant values $(p<0.01)$ are in bold.

\section{Conclusions}

Miller's classification of gingival tissue recessions was evaluated by 4 examiners using 200 photographs and yielded substantial to almost perfect agreement, with higher agreement for the anterior teeth. The present study offers the so far missing proof on the sufficient interand intra-observer agreement of this classification.

References

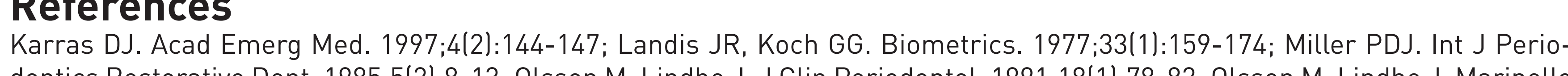
dontics Restorative Dent. 1985;5(2):8-13; Olsson M, Lindhe J. J Clin Periodontol. 1991;18(1):78-82; Olsson M, Lindhe J, Marinello
CP. J Clin Periodontol. 1993:20 (8):570-577. Seibert J, Lindhe J. Textbook of clinical periodontology Copenhagen. Munksgard, 1989:477-514. 